\title{
Enzyme structure dynamics of xylanase I from Trichoderma longibrachiatum
}

\author{
Ugur Uzuner ${ }^{1,2}$, Weibing Shi ${ }^{1,2}$, Lantao Liu ${ }^{1,2}$, Sanmin Liü 2,3 , Susie Y Dai ${ }^{3,4^{*}}$, Joshua S Yuan ${ }^{1,2,5^{*}}$ \\ From Seventh Annual MCBIOS Conference. Bioinformatics: Systems, Biology, Informatics and Computation \\ Jonesboro, AR, USA. 19-20 February 2010
}

\begin{abstract}
Background: Enzyme dynamics has recently been shown to be crucial for structure-function relationship. Among various structure dynamics analysis platforms, HDX (hydrogen deuterium exchange) mass spectrometry stands out as an efficient and high-throughput way to analyze protein dynamics upon ligand binding. Despite the potential, limited research has employed the HDX mass spec platform to probe regional structure dynamics of enzymes. In particular, the technique has never been used for analyzing cell wall degrading enzymes. We hereby used xylanase as a model to explore the potential of HDX mass spectrometry for studying cell wall degrading enzymes.

Results: HDX mass spectrometry revealed significant intrinsic dynamics for the xylanase enzyme. Different regions of the enzymes are differentially stabilized in the apo enzyme. The comparison of substrate-binding enzymes revealed that xylohexaose can significantly stabilize the enzyme. Several regions including those near the reaction centres were significantly stabilized during the xylohexaose binding. As compared to xylohexaose, xylan induced relatively less protection in the enzyme, which may be due to the insolubility of the substrate. The structure relevance of the enzyme dynamics was discussed with reference to the three dimensional structure of the enzyme. HDX mass spectrometry revealed strong dynamics-function relevance and such relevance can be explored for the future enzyme improvement.

Conclusion: Ligand-binding can lead to the significant stabilization at both regional and global level for enzymes like xylanase. HDX mass spectrometry is a powerful high-throughput platform to identify the key regions protected during the ligand binding and to explore the molecular mechanisms of the enzyme function. The HDX mass spectrometry analysis of cell wall degrading enzymes has provided a novel platform to guide the rational design of enzymes.
\end{abstract}

\section{Background}

Xylan is the major hemicellulose component of the plant cell wall and the second most abundant natural polysaccharide. Most of xylan is a heteropolysaccharide consisting of $\beta$-1,4-linked D-xylose monomers in connection with side branches of arabinosyl, glucuronosyl, acetyl, uronyl, and mannosyl residues [1]. Complete degradation of xylan structures requires the concerted and synergistic function of several enzymes including

\footnotetext{
* Correspondence: susie@otsc.tamu.edu; syuan@tamu.edu

'Department of Plant Pathology and Microbiology, Texas A\&M University,

College Station, TX 77843, USA

${ }^{3}$ Department of Veterinary Pathobiology, Texas A\&M University, College

Station, TX, 77843, USA

Full list of author information is available at the end of the article
}

endo- $\beta$-1, 4-xylanases (EC 3.2.1.8) [2]. Due to the broad applications in biopulping and biobleaching in paper industry, xylanase has been one of the major research focuses for bioconversion [1]. In particular, endoxylanases have been thoroughly studied as the major lignocellulosic biomass degradation enzymes. Xylanases with high substrate binding specificity, enhanced enzymatic activity, and increased thermostability are needed for various biorefinery applications. Tremendous efforts have been devoted to improve xylanase and cellulase enzyme performance by manipulating the protein amino sequence in the past [3]. However, sequence-based protein modification has its limitations $[4,5]$. It is experimentally infeasible to test all possible mutants of a 
protein, and it is time consuming since the majority of the manipulated sequences do not fold properly into functional proteins [6]. Suitable techniques thus are needed to guide enzyme improvement with structurefunction relationship for better enzyme rational design and engineering.

Structure dynamics has become an important consideration for enzyme engineering [7-14]. The structure dynamics during the enzyme catalysis can be derived from molecular dynamics simulations, NMR, and mass spectrometry-based methods. In particular, novel HDX mass spectrometry platforms provide the structure dynamics information for enzyme engineering $[15,16]$. Recent research unveiled how structure dynamics is related to enzyme function $[9,13,16]$. The structure dynamics-guided approach has been successfully used for enzyme activity improvement $[7,8]$. Hydrogen/deuterium exchange mass spectrometry (HDX-MS) represents one of the most widely used platforms for exploring protein conformational dynamics, folding, and binding [17-22]. HDX mass spectrometry has been broadly applied to study protein dynamics and structure, in particularly for the protein binding with ligands, substrates, DNA and other molecules [23-27]. Such analysis has enabled the illustration of the enzyme substrate interaction mechanism and the protein binding molecular determinants $[28,29]$.

The fundamental concept of HDX mass spectrometry analysis is based on the mass increase of a protein when the protein protons exchange with the solvent deuterium [30]. The rate and percentage of the H/D exchange can be measured by mass to charge ratio $(\mathrm{m} / \mathrm{z})$ of the protein. The HDX mass spectrometry can be used to study the global and regional protein conformational changes with different platforms [31,32]. Coupled with protein digestion and chromatography separation, the HDX mass spectrometry is able to profile different regions of protein for H/D exchange based on the peptide H/D exchange rate and percentage. The underlying cause of HDX structure dynamics may involve changes in hydrogen bonds and other forces [27]. For instance, if the protein binding with ligand leads to more H/D exchange in a region, the ligand binding is expected to induce conformational changes to destabilize the region. HDX mass spectrometry thus allows us to probe the protein structure dynamics changes during enzymatic reactions $[9,28,33]$.

The HDX platform comes with the advantages of mass spectrometry analysis: fast, straightforward, and environmentally friendly [27]. The HDX mass spectrometry technologies thus provide user-friendly alternatives to study the structure and dynamics of xylanase in a way that is not possible with other technologies. The advantages of HDX mass spectrometry over X-Ray and
NMR are higher throughput, less protein purity requirements, and the dynamics and stability information rendered $[34,35]$. Compared with X-Ray or NMR techniques, HDX mass spectrometry is difficult to resolve structures at a single amino acid residue resolution. The resolution of the techniques relies on the protease digestion, which will produce peptides of varied length in a protein-dependent manner. In addition, the conformational changes observed are the backbone changes, and the side chain information is limited. However, it is of great interest to resolve peptide regions that span several amino acid residues to localize the stabilized or destabilized region during the catalysis or inhibition. The backbone changes contribute to the conformational changes involved in enzyme reactions $[24,28]$. The combination of HDX mass spectrometry with X-ray data and computational modelling is a potent way to provide more detailed information regarding the structure, stability, and dynamics of enzyme/substrate or enzyme/inhibitor interactions.

In the present study, the structure dynamics changes of the Trichoderma longibrachiatum xylanase enzyme upon binding with xylohexaose and xylan ligands was evaluated with HDX mass spectrometry analysis. The analyses revealed important regional dynamics of xylanase upon ligand binding. Combination of the structure dynamics data from the HDX analyses and the static structure information from the X-ray crystallography provided novel insights for further enzyme improvement.

\section{Results and discussion}

\section{Data processing for HDX mass spectrometry}

The xylanase enzyme of $T$. longibrachiatum shared $100 \%$ sequence identity to that of $T$. reesei. A total of 57 digested peptides were identified in the MSMS data acquisition (Additional file 1) with the sequence coverage of $91 \%$ (Figure 1). In addition, the HDX analyses rendered 45 peptides with significant signal to noise ratio $(S / N>3)$ and gave $71 \%$ sequence coverage. The covered region is given in Figure 2. Twelve peptides that were identified in the MSMS acquisition could not be measured accurately in the HDX experiments due to co-elution problems or weak signal after long exchange times (for precise description, see Additional file 1). The representative deuterium incorporation spectra were illustrated in Figure 3 for peptide 'YTIWENTRVNEPSIQGTAT' (residues; 102-120).

\section{Structure dynamics revealed by HDX analysis in Apo protein}

The HDX analysis of apo xylanase showed that the enzyme dynamics is regional specific (Figure 2). Regions that include residues 77 to 101 and 107 to 121 showed the greatest hydrogen deuterium exchange in the apo 


\begin{tabular}{|c|c|c|c|c|c|c|c|c|c|c|}
\hline 5 & 10 & 15 & 20 & 25 & 30 & 35 & 40 & 45 & 50 & 55 \\
\hline 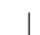 & $\mid$ & | & 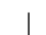 & 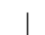 & | & 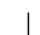 & 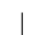 & 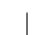 & | & 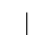 \\
\hline
\end{tabular}

ASINYDQNYQT GGQVSYSPSNTGFSVNWNT QDDFVVGVGWT T GSSAPINFGGSFSVNSGT

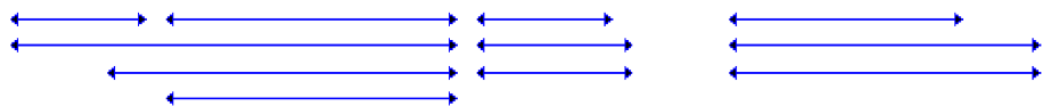

\begin{tabular}{|c|c|c|c|c|c|c|c|c|c|c|}
\hline 65 & 70 & 75 & 80 & 85 & 90 & 95 & 100 & 105 & 110 & 115 \\
\hline 1 & 1 & 1 & 1 & 1 & 1 & 1 & 1 & 1 & 1 & 1 \\
\hline
\end{tabular}

GLLSVYGWSTNPLVEYYI MEDNHNYPAQGTVKGTVTSDGATYTI WENTRVNEPSI QGTAT

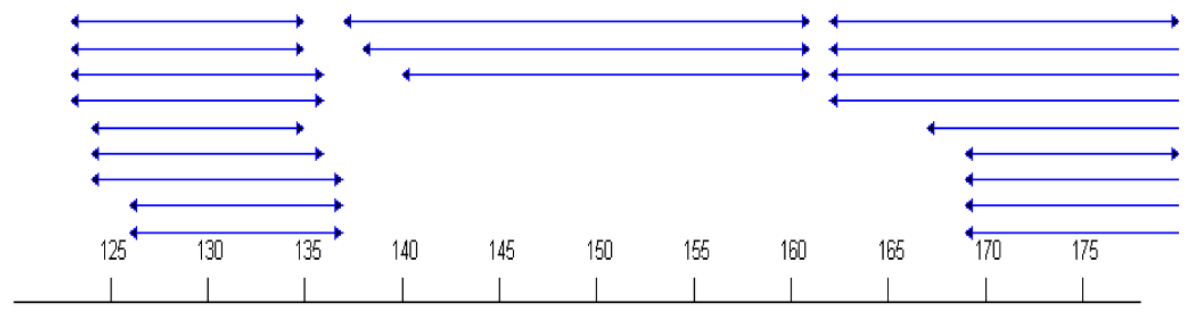

FNQYI SVRNSPRTSGTVTVQNHFNAWASLGLHLGQMNYQVVAVEGWGGSGSASQSVSN

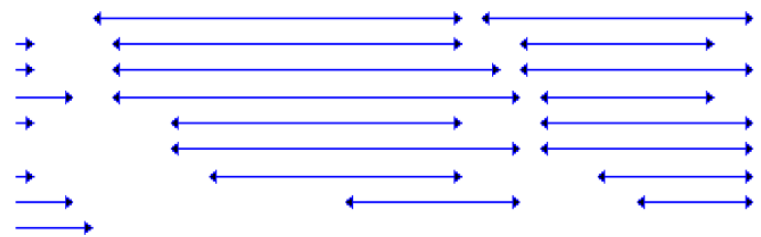

Figure 1 Peptides analyzed in the HDX experiment for xylanase (1XYN chain A). The blue arrowed lines donate the sequence of the peptide.

enzyme, and the $\mathrm{N}$-terminal of the enzyme is less dynamic according to the overall hydrogen deuterium exchange. Overall, the enzyme is dynamic based on the HDX experiment considering that the average exchange percentage is $50 \%$ for all five exchange times (i.e. 60 , 240, 960, 1920, and 3840 seconds).

\section{Differential HDX analysis of xylanase}

Differential HDX analysis of the apo protein versus the protein ligand complex revealed the structure dynamics change of the enzyme upon ligand binding. Figure 4 gave two examples that regions were protected in the HDX experiment. The protected region had less deuterium incorporation with the same exchange time. Peptide 'VGWTTGSSAPINF' in Figure 4A (residues; 38-50) and peptide 'YTIWENTRVNEPSIQGTAT' in Figure 4B (residues; 102-120) showed significant protection in the HDX measurements. Contrary to that, peptide
'SVYGWSTNPLVEY' (residues; 64-76), and peptide 'SVYGWSTNPLVEYY' (residues; 64-77) showed similar exchange pattern in the apo protein and holo protein, which suggested those regions had little structure dynamics change upon xylohexaose binding. More importantly, the two peptides had only one amino acid differences and had an essentially similar HDX profile, which highlighted the reproducibility of our data. The differences in the structure dynamics probed by hydrogen deuterium exchange potentially reflected the differential local structure protections. The common speculation of mechanism for hydrogen/deuterium exchange involves hydrogen bonding and possible solvent accessibility, which lead to different hydrogen/deuterium exchange rates in different protein regions [36]. Nevertheless, the HDX analysis revealed the differential structure dynamic of different xylanase regions when binding with substrate xylohexaose. 


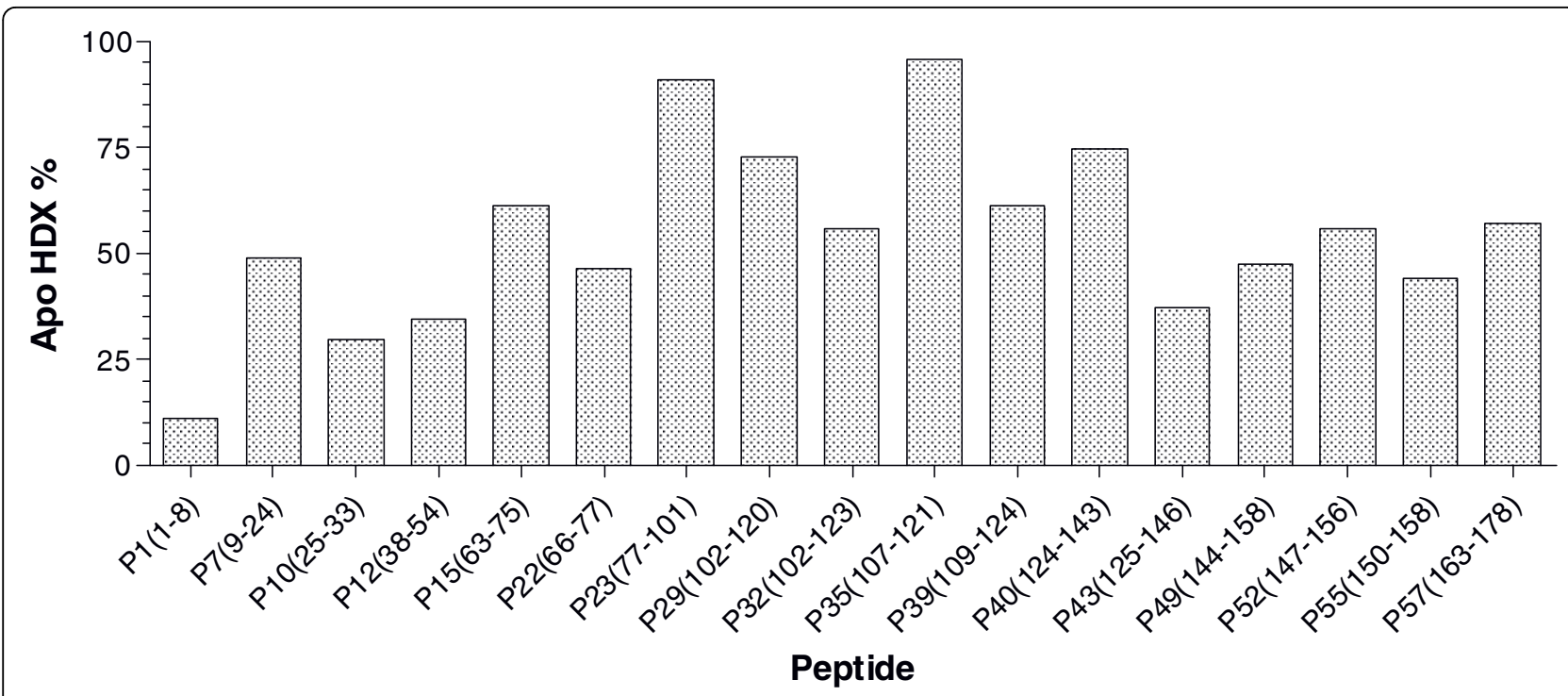

Figure 2 HDX percentage of apo xylanase for representative peptides. The selected peptides have a total of $91 \%$ sequence coverage. The percentage is the averaged value of five HDX experiments with different exchange times (60,240, 960, 1920, and 3840 seconds).

\section{Statistical analysis for differential HDX data}

Despite the visualization in Figure 4, the differential HDX profile needs to be determined statistically. We chose the last three time points for the paired student $t$ test analysis to compare the apo and ligand binding mass spectra centroid value as shown in Additional file 1 . For the apo enzyme, the maximum $\mathrm{D}_{2} \mathrm{O}$ exchange percentage (HDX percentage) for each peptide ranged from $10 \%$ to $95.7 \%$. In parallel, HDX percentage was also defined for protein binding with the substrate. The level of protection for a particular peptide can be determined by two methods. First, we can calculate the differences for the HDX percentage between the apo and holo protein. Second, we can calculate the differences of the centroid of the mass spectra for the peptides. The advantage for the percentage calculation is that it gives a numeric value that is normalized against the size and the charge state of the protein. The advantage of centroid value is that it is the direct measurement of the HDX mass spectra and thus can be more readily subject to various statistical analyses. We therefore combined the strength of the two types of measurement. For the visualization in Figure 5, we adopted the percentage differences. For the statistical analysis in Additional file 1, we included the paired student's t-test of mass spectra centroid for peptides to compare the apo and holo proteins.

The results revealed that the substrate xylohexaose played an important role in the stabilization of the xylanase chain A, because the HDX rate for most of the peptides were smaller than apo, and the centroid values were significantly lower than those of apo protein for most peptides $(\mathrm{P}<0.01)$. Only a few peptides such as the peptide 'SVNWNTQDD' and 'NTRVNEPSIQGTATF' showed no significant differences in peptide centroid between the apo and holo protein $(t=0.9553$ and $0.9681, P=0.3627$ and 0.3614 ).

In contrast, most of the peptides were not significantly stabilized by xylan binding. Upon the xylan binding, only a few peptides including 'SVNWNTQDD' $(t=4.76, P<0.01)$, 'LSVYGWSTNPLVEY' $(t=3.08, P=0.02)$, 'YGWSTNPLVEYY' $(t=4.52, P<0.01)$, 'ISVRNSPRTSGTVTVQNHF' $(t=3.23, P=0.01)$, and 'NAWASLGLHLGQMNY' $(t=2.81$, $P=0.02)$ was significantly stabilized by xylan as shown by the statistical test in Additional file 1. Even though some peptide exhibited large HDX percentage differences, these peptides did not show significant differences in the centroid values. The results highlighted the importance of statistical analysis in the interpretation of structure dynamics.

\section{Correlation of HDX data with X-ray structure}

Correlation of the HDX data with the X-ray three dimensional structure data can also provide useful information to understand the enzyme function. The 3D structure of xylanase from T. reesei was used as the template to overlay the structure dynamics with the static structure, because the enzyme shares $100 \%$ amino acid sequence identity with the T. longibrachiatum Xylanase. Excellent consistency between the X-ray structure and the HDX analyses was observed. The active sites of XYN1 Glu75, Glu164 are located in B6 and B4 $\beta$ strands [37]. The protein was described to have the analogy of the right hand. As shown in Figure 5A, the HDX profile 


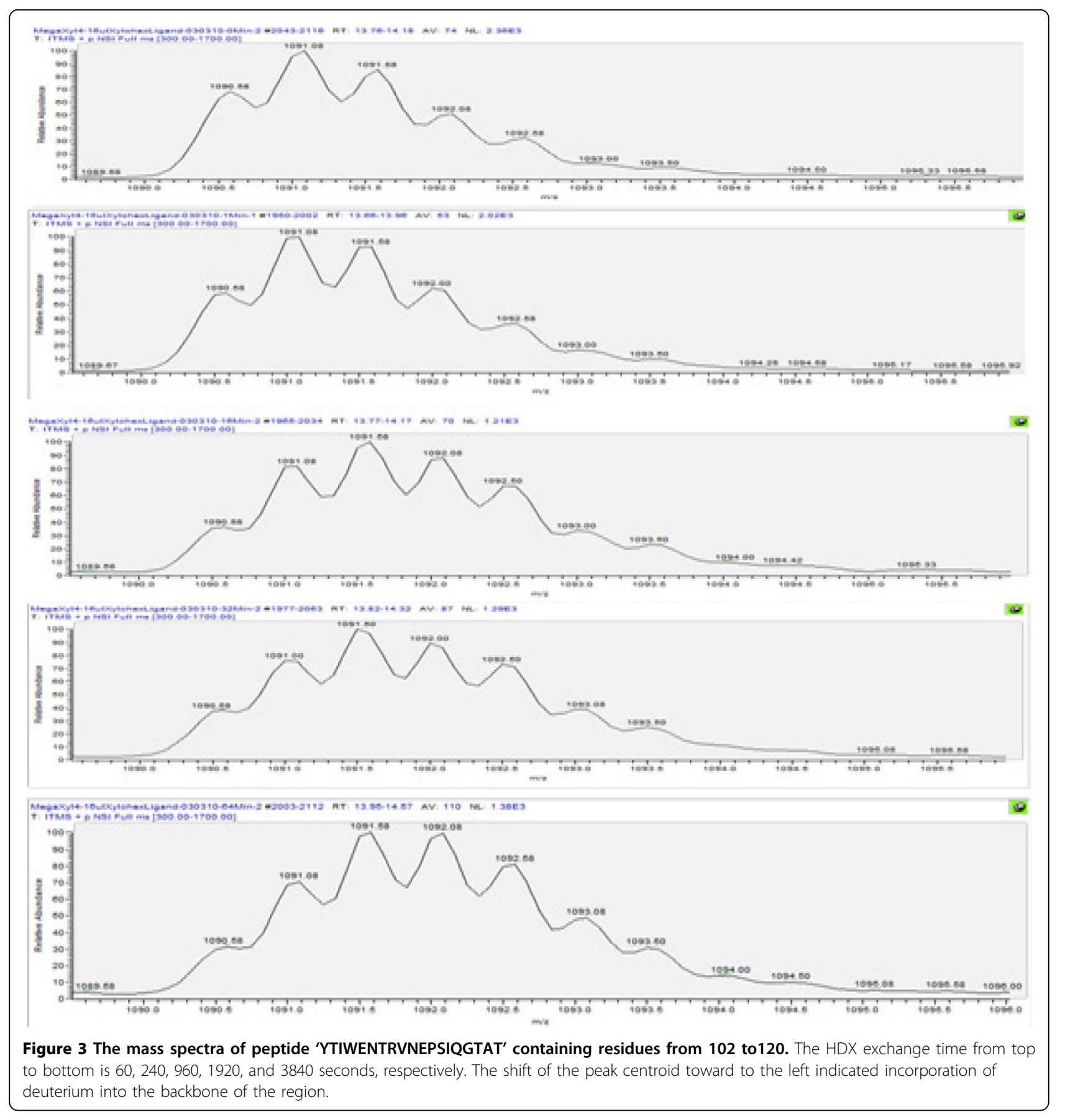

revealed that the regions close to the reaction center had the highest protection when the enzyme bound with xylohexaose ligand. Considering that enzyme reaction often involves the substrate interaction with the reaction sites, leading to the stabilization in the region, the results highlighted the potential regions essential for the ligand binding process and substrate specificity (Figure $5 \mathrm{~A}$ ). In addition, the thumb region of the protein as suggested in the previous publication [37] was highly stabilized during the ligand binding. The stabilization indicated that the thumb region of xylanase might also play an important role in binding and processing of the xylohexaose substrates. HDX analysis thus had the potential to reveal important regions for substrate binding and enzyme reaction beyond the reaction sites. Besides the thumb regions, we also identified some other stabilized regions outside of the substrate binding groove. For instance, the peptide containing residues 

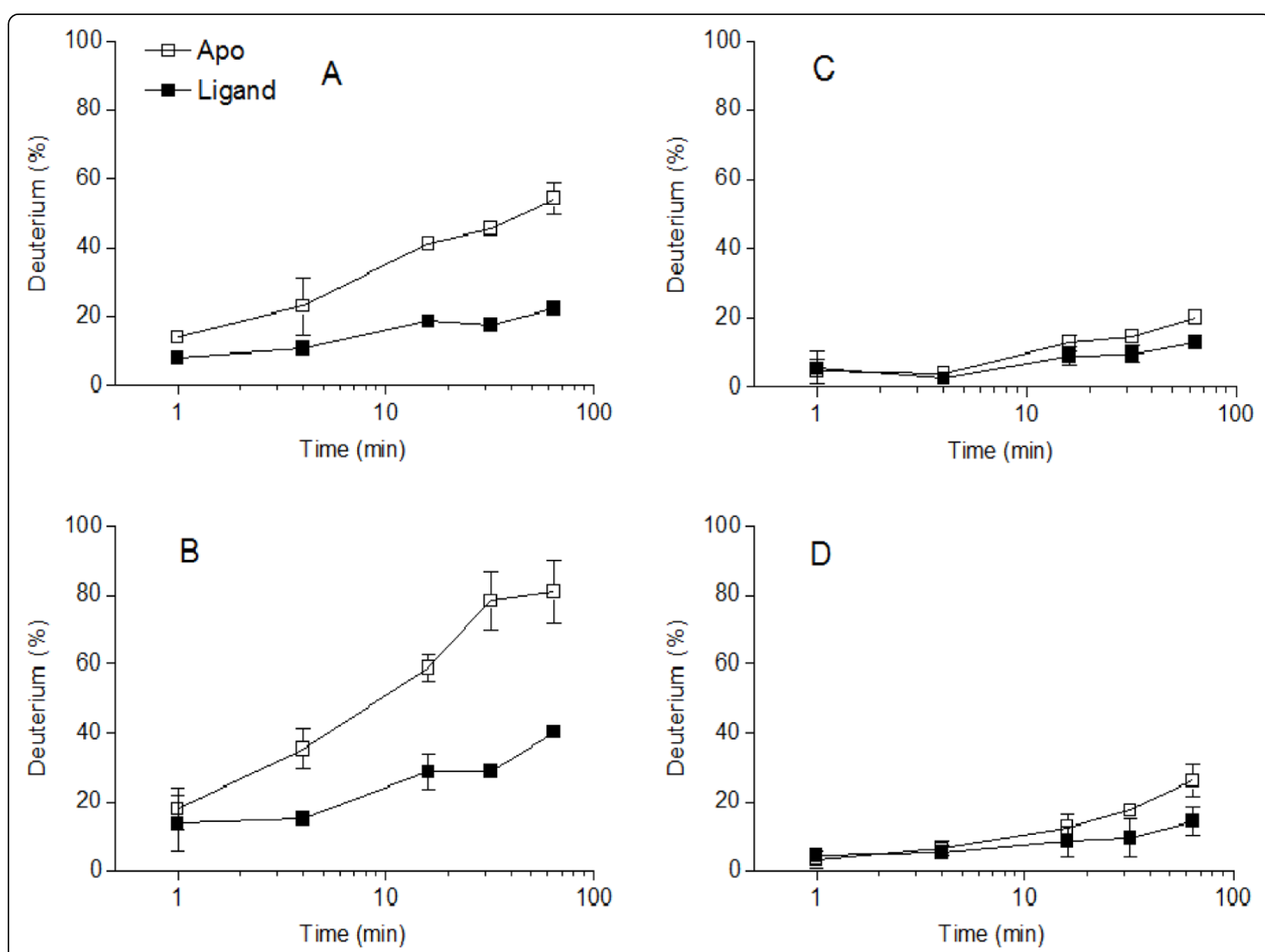

Figure 4 Deuterium incorporation curves of four peptides. HDX data for the apo xylanase is represented by the open square, and HDX data for the xylanase interacting with xylohexaose substrate is represented with the closed square. A. HDX data for peptide 'VGWTTGSSAPINF' containing residues from 38 to 50. B. HDX data for peptide 'YTIWENTRVNEPSIQGTAT' containing residues from 102 to 120. C. HDX data for peptide 'SVYGWSTNPLVEY' containing residues from 64 to 76, D. HDX data for peptide 'SVYGWSTNPLVEYY' containing residues from 64 to 77.

from 77 to101 located only one residue away from one of the active sites and exhibited the greatest protection in the HDX experiment. The protection was also observed with the peptide containing residues from 6 to 24 , which consists one of the fingers of the right hand structure of the protein.

\section{Comparative analysis of structure dynamics when binding with two substrates}

As aforementioned, we analyzed the HDX profile for two substrates in our study, xylan and xylohexaose. The results showed rather different structure dynamics induced by different substrate binding as shown Figure 5 and Additional file 1. Overall, there seemed to be less enzyme structure dynamics changes for xylan binding than those for xylohexaose binding. Even though the differences in HDX percentage as shown in Figure 5B still showed some regions of changes, most of peptide did not show a statistically significant change in the HDX percentage. The results along with the HDX profile indicated a high variation for the HDX percentage measurement. Such variation between the substrates is due to the solubility of the xylan. Xylan is not entirely soluble and the solution forms an emulsion during the experiment, which might have led to the significant variation of the HDX analysis during the xylan binding experiments. The results indicated the xylohexaose binding might have rendered much more reliable data for mechanism studies.

\section{HDX profile and enzyme improvement for the future}

Engineering protein flexibility (plasticity) can be used to enhance substrate/ligand specificity of the protein by increasing the rigidity of flexible residues [38]. For this reason, we carried out the HDX analysis for xylanase to identify the critical sequences that could be stabilized to 

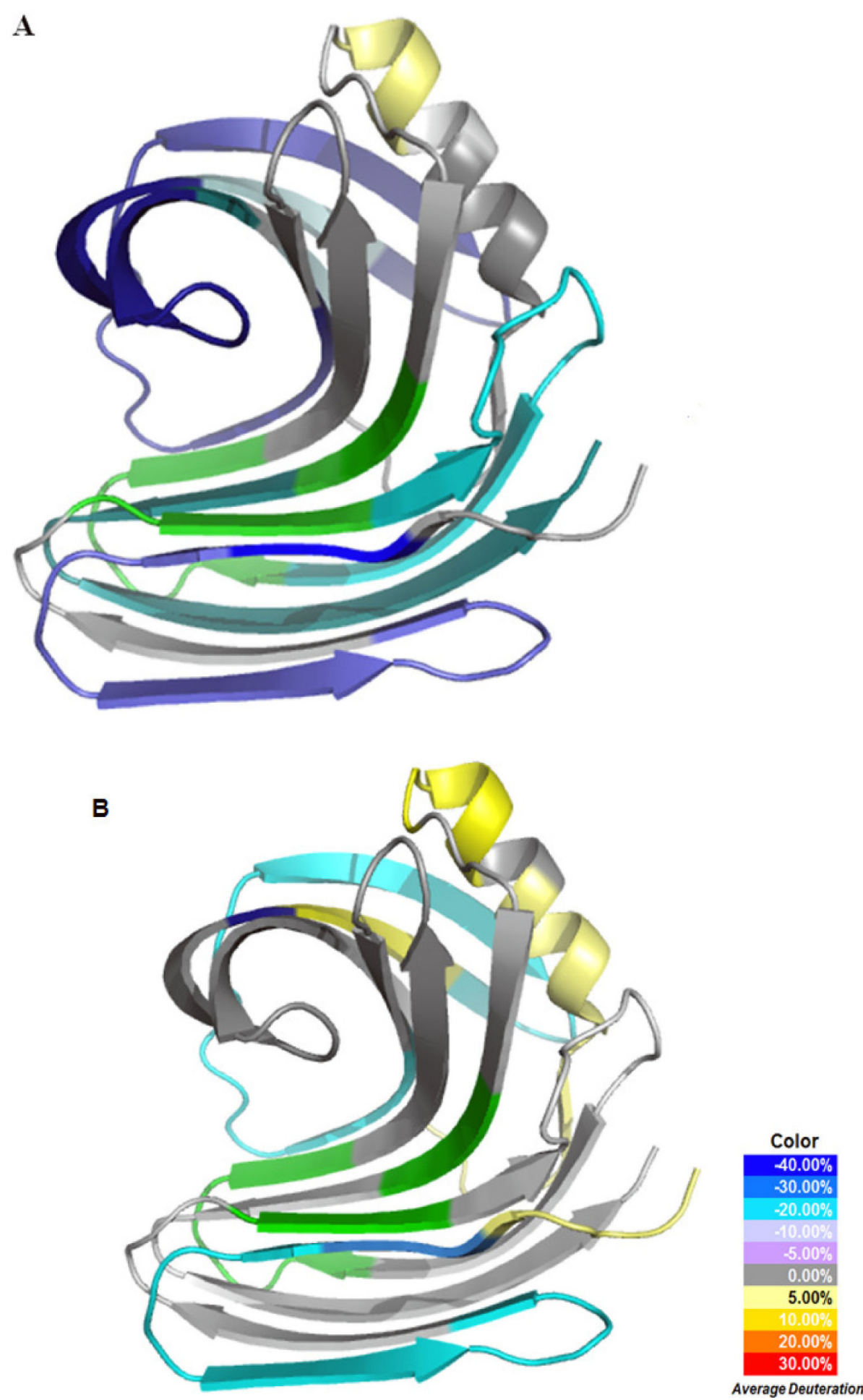

Figure 5 HDX profile overlaid onto xylanase crystal structures. A) HDX profile of xylanase/xylohexaose complex overlaid onto the apo xylanase crystal structure (PDB: 1XYNA), B) HDX profile of xylanase/xylan complex overlaid onto apo xylanase crystal structure (PDB:1XYNA), The color legend shows the deuterium incorporation difference by subtracting deuterium incorporation content of holo xylanase from apo xylanase. The green coloured regions represent peptides that are not detected after pepsin digestion or cannot be measured accurately in the HDX experiments due to co-elution problems. 
strengthen the local interactions of the groove region and neighboring residues. Our data provided important information regarding the global and local dynamics of xylanase enzymes when binding with different substrates. We aim to use the structure dynamics information to guide future enzyme engineering. The highly stabilized regions are believed to be important for enzyme-substrate interaction and thus are the regions of choice for downstream protein engineering work.

In order to relay the HDX analysis result to enzyme evolution and improvement, we carried out the multiple sequence alignment of xylanase enzymes from different species and families as shown in Figure 6. Ten fungal and two bacterial xylanase 1 sequences were aligned together with the $T$. longibrachiatum xylanase. The multiple sequence alignment revealed that some highly conserved regions like peptide 'RVNEPSIQGTATFNQY' (residues; 109-124) corresponded to the regions significantly stabilized during the substrate binding. This peptide consists of the thumb region of the xylanase and locates very close to the active site (Glu75) of the protein (Figure 5A and 5B; Figure 6). The significant protection indicated its crucial role in substrate recruitment or holding. The evolutionary evidence and structure dynamics information correlated with one another indicating the region to be one of the structure dynamics determinants for enzyme function. Besides the peptide containing residues from 109 to 124, many other regions were shown to be significantly protected during the substrate binding, and some of these regions were less conserved. We are designing domain swapping experiments to modify various regions for enzyme improvement and mechanistic study.

In industrial applications, the hydrolysis step of the lignocellulosic biomass processing requires unique functions of enzymes that can be highly efficient with extreme $\mathrm{pH}$ tolerance, increased thermostability, and improved substrate specificity. HDX mass spectrometry provided a powerful platform to study enzyme dynamics at different conditions, and thus could be used to guide the enzyme improvement for various features. We are using the HDX analysis to explore a broader range of cell wall degrading enzymes for both mechanistic study and enzyme engineering.

\section{Conclusions}

Overall, HDX analysis has revealed significant intrinsic dynamics for xylanase enzyme and such dynamics might be important for the enzyme function. Specifically, different regions of the apo xylanase showed a differential HDX rate. The substrate binding leads to significant protection or stabilization effects, where some regions near the reaction sites were significantly stabilized by the enzyme substrate xylohexaose. The xylohexaose binding also induced protection on regions beyond the reaction center. In contrary to xylohexaose, xylan binding induced fewer changes in enzyme structure dynamics, assumingly due to the insufficient binding resulted from the insolubility of xylan. The structure dynamics information for substrate binding indicated that many regions in the protein coordinatively changed conformation to fulfil the function of substrate docking and catalysis. The structure dynamics information also correlated with the enzyme evolution to a certain degree, where the evolutionarily conserved regions were very well protected by the substrate binding. These regions were expected to be essential for the enzyme function. Overall, HDX mass spec analysis allows us to identify the novel structure determinants for the enzyme function that could not be found with traditional X-ray or NMR techniques. HDX mass spectrometry thus provided a novel platform to guide the rational design of enzymes.

\section{Material and methods}

\section{Protein and reagents}

The endo-1,4- $\beta$-Xylanase M2 (EC 3.2.1.8) of T. longibrachiatum was purchased from Megazyme (Megazyme International Ireland Ltd., Wicklow, Ireland) and was used throughout the all HDX experimental processes without further purification. The protein solution was provided as a mixture of ammonium sulphate $45 \%$, sodium azide $0.02 \%$, water $45 \%$ and Xylanase M2 ( $T$. longibrachiatum) $2.5 \%$. The substrate, xylohexaose (molecular weight, MW: $810.70 \mathrm{~g}^{-1}$, Cat No: O-XHE) with $>95 \%$ purity, was also provided from Megazyme. The second substrate of xylanase enzyme for this study was $2 \%$ oats spelts xylan obtained from TCI AMERICA (Portland, OR, Cat No: X0011).

\section{HDX experiment}

HDX experiments were similar to those previously described except without using the LEAP Technologies Twin HTS PAL liquid handling robot $[39,40]$. Briefly, the xylanase protein solution was used without further purification at the concentration of $13.6 \mathrm{mg} / \mathrm{mL}$ in solution. The xylohexaose was dissolved in a $\mathrm{D}_{2} \mathrm{O}$ buffer (20mM Tris-HCL, $100 \mathrm{mM} \mathrm{KCL}$, and $1 \mathrm{mM}$ DTT in $\left.\mathrm{D}_{2} \mathrm{O}, \mathrm{pD} 7.9\right)$ to reach a final concentration of $25 \mathrm{mM}$. Xylan was dissolved in $\mathrm{D}_{2} \mathrm{O}$ buffer to make up a $1 \%$ solution. Four $\mu \mathrm{L}$ of the xylanase solution was mixed with $16 \mu \mathrm{L}$ of the ligand $\mathrm{D}_{2} \mathrm{O}$ buffer for HDX experiments at room temperature for $0,60,240,960,1920$, and 3840 seconds, respectively. After the incubation in $\mathrm{D}_{2} \mathrm{O}$ at an aforementioned hydrogen deuterium exchange time, the exchange reaction was quenched with $30 \mu \mathrm{L}$ ice-cold solution containing $2 \mathrm{M}$ urea and $1 \%$ Trifluoroacetic acid (TFA), injected into an injection 


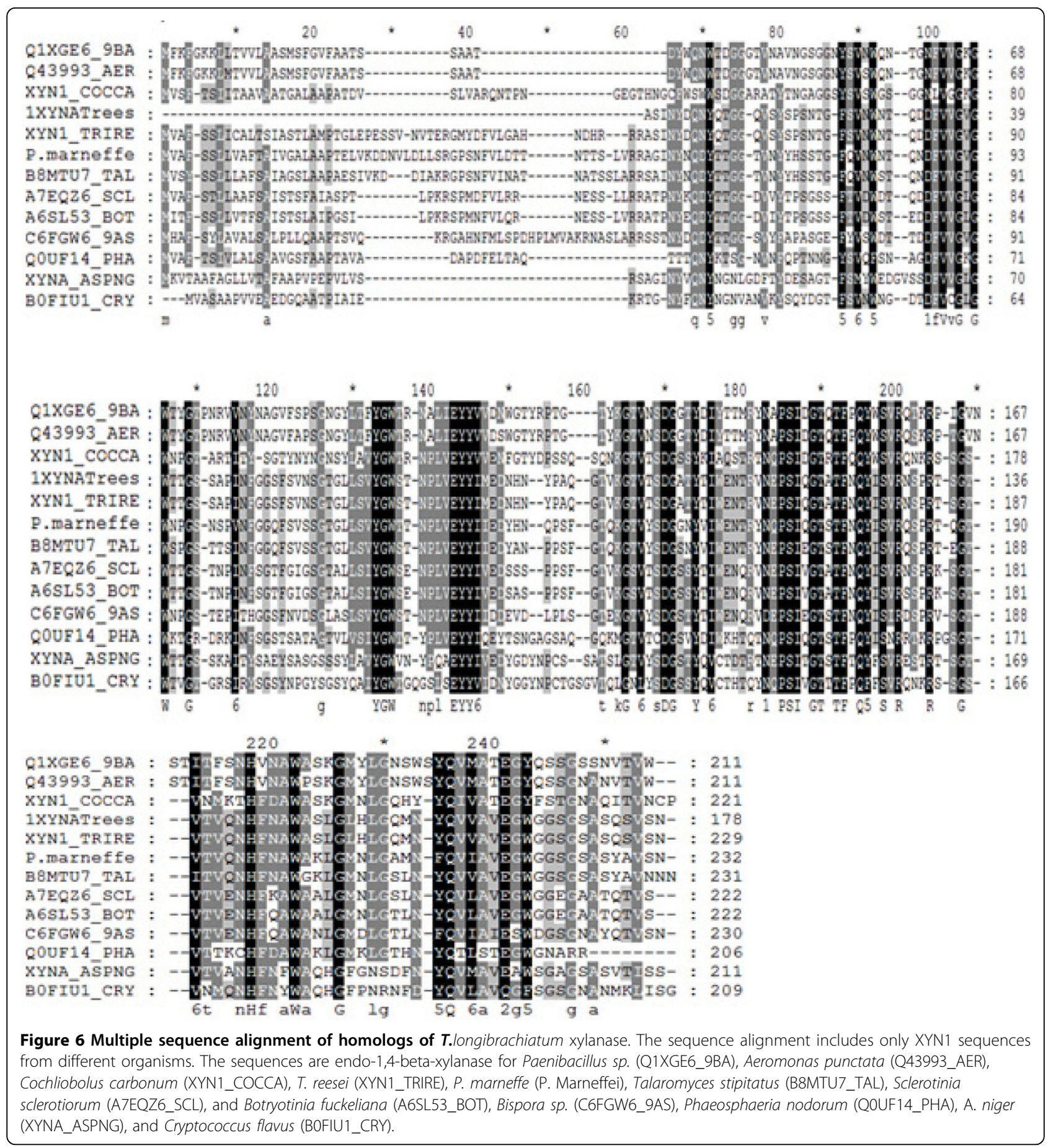

valve with $50 \mu \mathrm{L}$ sample loop, and then passed through a pepsin column (Applied biosystems, Foster City, CA) by a solvent pump (0.1\% TFA in water) with flow rate at $200 \mu \mathrm{L} / \mathrm{min}$. The pepsin column was kept on ice. The digested xylanase peptides were then eluted through a micro peptide cartridge (Michrom Bioresources, Inc., Auburn, CA) and desalted. The digestion and desalting takes a total of $5 \mathrm{~min}$. Peptides were then eluted across a $2.1 \mathrm{~mm} \times 5 \mathrm{~cm}$ C18 column (Thermo Scientific, Waltham, MA) with a linear gradient of $2 \%-50 \%$ Solution B over Solution A for 10 min (Solvent A, 0.1\% formic acid in water; solvent $\mathrm{B}, 0.1$ formic acid $80 \%$ acetonitrile, $20 \%$ water; flow rate $200 \mu \mathrm{L} / \mathrm{min}$ ). Mass spectrometric analyses were carried out with the capillary temperature at $280{ }^{\circ} \mathrm{C}$ using LC-LTQ mass spectrometer (Thermo Scientific, Waltham, MA). The apo xylanase HDX 
experiment was performed with the same protocol except that the $\mathrm{D}_{2} \mathrm{O}$ solution contained no ligand.

\section{Peptide identification and HDX data processing}

Product ion spectra were acquired in a data-dependent MS/MS mode. The precursor ion survey scan was performed and the five most abundant ions were selected for product ion analysis. MS/MS *.raw data was first converted into *.MS2 file and then searched against the database containing xylanase using SEQUEST algorithm (Bioworks, Thermo Finnigan, CA). All peptide ion assignments were inspected manually.

The weighted average $\mathrm{m} / \mathrm{z}$ values of each peptide ion isotopic cluster were calculated with the in-house developed software named as HDXanalyzer (manuscript in preparation). The deuteration level was calculated based on the following equation, and corrections for back-exchange were made based on $70 \%$ deuterium recovery and accounting for $80 \%$ deuterium content in the ion-exchange buffer.

$$
\text { Deuterationlevel }(\%)=\frac{m / z(P)-m / z(N)}{m / z(F)-m / z(N)} * 100
$$

where $\mathrm{m} / \mathrm{z}(\mathrm{P}), \mathrm{m} / \mathrm{z}(\mathrm{N})$, and $\mathrm{m} / \mathrm{z}(\mathrm{F})$ are the centroid value of partially deuterated peptide, nondeuterated peptide, and fully deuterated peptide, respectively [41].

\section{Additional material}

Additional file 1: HDX analyses of peptides and statistical analysis.

\section{Acknowledgements}

The research was supported by the South Central Region Sungrant (Oklohoma State University subaward No: AB-5-61770.2 TEAS 6), the start up fund for Joshua Yuan from the Department of the Plant Pathology and Microbiology, and the start up fund for Susie Dai from the Veterinary Pathobiology at Texas A\&M University. In addition, the Texas Agrilife Bioenergy Research Initiative funding has also helped to support material and supplies. Ugur Uzuner was also a recipient of the government fellowship from The Ministry of National Education of the Republic of Turkey. We appreciate Ryan Syrenne for editing the article.

This article has been published as part of BMC Bioinformatics Volume 1 Supplement 6, 2010: Proceedings of the Seventh Annual MCBIOS Conference. Bioinformatics: Systems, Biology, Informatics and Computation. The full contents of the supplement are available online at http://www.biomedcentral.com/1471-2105/11?issue=S6.

\section{Author details \\ ${ }^{1}$ Department of Plant Pathology and Microbiology, Texas A\&M University, College Station, TX 77843, USA. ${ }^{2}$ Institute for Plant Genomics and Biotechnology, Texas A\&M University, College Station, TX 77843, USA. ${ }^{3}$ Department of Veterinary Pathobiology, Texas A\&M University, College Station, TX, 77843, USA. ${ }^{4}$ Office of the Texas State Chemist, Texas A\&M University, College Station, TX 77843, USA. ${ }^{5}$ Advanced Research Institute of Sustainable Energy, Texas A\&M University, College Station, TX, 77843, USA.}

\section{Authors' contributions}

Joshua Yuan and Susie Dai designed the experiments. Ugur Uzuner performed the experiments. Ugur Uzuner, Weibing Shi, Lantao Liu, and
Sanmin Liu performed the data analysis. Ugur Uzuner, Susie Dai, and Joshua Yuan wrote the manuscript.

\section{Competing interest}

The authors have no competing interest for this article.

Published: 7 October 2010

\section{References}

1. Tsujibo H, Ohtsuki T, lio T, Yamazaki I, Miyamoto K, Sugiyama M, Inamori Y: Cloning and sequence analysis of genes encoding xylanases and acetyl xylan esterase from Streptomyces thermomviolaceus OPC-520. Appl. Environ Microbiol 1997, 63:661-664.

2. Jun H, Bing Y, Keying Z, Xuemei D, Daiwen C: Thermostable carbohydrate binding module increases the thermostability and substrate-binding capacity of Trichoderma reesei xylanase 2. New Biotech 2009, 26:53-59.

3. Barak Y, Nov Y, Ackerley DF, Matin A: Enzyme improvement in the absence of structural knowledge: a novel statistical approach. The ISME J 2008, 2:171-179.

4. Dennett DC: Darwin's dangerous idea: Evolution and the meanings of life. Simon \& Schuster Inc New York, NY 1995.

5. Chatterjee R, Yuan L: Directed evolution of metabolic pathways. Trends Biotech 2006, 24:28-38.

6. Arnold FH: Fancy footwork in the sequence space shuffle. Nat Biotech 2006, 24:328-330.

7. Agarwal PK: Role of protein dynamics in reaction rate enhancement by enzymes. J Am Chem Soc 2005, 127(43):15248-15256.

8. Agarwal PK: Enzymes: An integrated view of structure, dynamics and function. Microb Cell Fact 2006, 5.

9. Henzler-Wildman K, Kern D: Dynamic personalities of proteins. Nature 2007, 450(7172):964-972

10. Liu YH, Konermann L: Enzyme conformational dynamics during catalysis and in the 'resting state' monitored by hydrogen/deuterium exchange mass spectrometry. FEBS Lett 2006, 580(22):5137-5142.

11. Eisenmesser EZ, Millet O, Labeikovsky W, Korzhnev DM, Wolf-Watz M, Bosco DA, Skalicky JJ, Kay LE, Kern D: Intrinsic dynamics of an enzyme underlies catalysis. Nature 2005, 438(7064):117-121.

12. Daniel RM, Dunn RV, Finney JL, Smith JC: The role of dynamics in enzyme activity. Annu Rev Biophys Biomol Struct 2003, 32:69-92.

13. Eisenmesser EZ, Bosco DA, Akke M, Kern D: Enzyme dynamics during catalysis. Science 2002, 295(5559):1520-1523.

14. Hammes-Schiffer S, Benkovic SJ: Relating protein motion to catalysis. Annu Rev Biochem 2006, 75:519-541.

15. Maity H, Lim WK, Rumbley JN, Englander SW: Protein hydrogen exchange mechanism: local fluctuations. Protein Sci 2003, 12(1):153-160.

16. Chalmers MJ, Busby SA, Pascal BD, He Y, Hendrickson CL, Marshall AG, Griffin PR: Probing protein ligand interactions by automated hydrogen/ deuterium exchange mass spectrometry. Anal Chem 2006, 78(4):1005-1014

17. Bae E, Phillips GN: Roles of static and dynamic domains in stability and catalysis of adenylate kinase. Proc Natl Acad Sci U S A 2006, 103(7):2132-2137.

18. Konermann $L$, Tong $X$, Pan $Y$ : Protein structure and dynamics studied by mass spectrometry: H/D exchange, hydroxyl radical labeling, and related approaches. J. Mass Spec 2008, 43:1021-1036.

19. Kaltashov IA, Eyles SJ: Mass spectrometry in biophysics. John Wiley \& Sons: Hoboken 2005.

20. Pan JX, Rintala-Dempsey A, Li Y, Shaw GS, Konermann L: Folding kinetics of the S100A11 protein dimer studied by time-resolved electrospray mass spectrometry and pulsed hydrogen-deuterium exchange. Biochemistry 2006, 45:3005.

21. Eyles SJ, Kaltashov IA: Methods to study protein dynamics and folding by mass spectrometry. Methods 2004, 34:88.

22. Miranker A, Robinson CV, Radford SE, Aplin R, Dobson CM: Detection of transient protein folding populations by mass spectrometry. Science 1993, 262:896

23. Powell KD, Ghaemmaghami S, Wang MZ, Ma L, Oas TG, Fitzgerald MC: A general mass spectrometry-based assay for the quantitation of proteinligand binding interactions in solution. J Am Chem Soc 2002, 124:10256-10257. 
24. Li J, Lim MS, Li S, Brock M, Pique ME, Woods VL, Craig L: Vibrid cholerae toxin-coregulated pilus structure analyzed by hydrogen/deuterium exchange mass spectrometry. Structure 2008, 16(1):137-148.

25. Brock M, Fan F, Mei FC, Li S, Gessner C, Woods VL, Cheng X: Conformational analysis of Epac activation using amide hydrogen/ deuterium exchange mass Spectrometry. J Biol Chem 2007, 282(44):32256-32263.

26. Lambris JD, Sfyroera G, Schuster M, Chen H, Tzekou A, Papp K, Winters M, Woods VL: Studies on the solvent accessibility of native $\mathrm{C} 3$ and its fragments, as analyzed by HDX-MS. Mol Immun 2007, 44(1-3):202-202.

27. Eyles SJ, Kaltashov IA: Methods to study protein dynamics and folding by mass spectrometry. Methods 2004, 34(1):88-99.

28. Englander SW: Hydrogen exchange and mass spectrometry: A historical perspective. J Am Soc Mass Spectrom 2006.

29. Begley MJ, Taylor GS, Brock MA, Ghosh P, Woods VL, Dixon JE: Molecular basis for substrate recognition by MTMR2, a myotubularin family phosphoinositide phosphatase. Proc Natl Acad Sci U S A 2006, 103(4):927-932.

30. Derunes C, Burgess R, Iraheta E, Kellerer R, Becherer K, Gessner CR, Li S, Hewitt K, Vuori K, Pasquale EB: Molecular determinants for interaction of SHEP1 with Cas localize to a highly solvent-protected region in the complex. FEBS Lett 2006, 580(1):175-178.

31. Roder $\mathrm{H}$, Elove GA, Englander SW: Structural characterization of folding intermediates in cytochrome $\mathrm{c}$ by $\mathrm{H}$-exchange labelling and proton NMR. Nature 1988, 335(6192):700-704.

32. Black BE, Foltz DR, Chakravarthy S, Luger K, Woods VL, Cleveland DW: Structural determinants for generating centromeric chromatin. Nature 2004, 430(6999):578-582.

33. Powell KD, Fitzgerald MC: High-throughput screening assay for the tunable selection of protein ligands. J Combin Chem 2004, 6(2):262-269.

34. Iyer GH, Garrod S, Woods VL, Taylor SS: Catalytic independent functions of a protein kinase as revealed by a kinase-dead mutant: Study of the Lys72 His mutant of cAMP-dependent kinase. J Mol Biol 2005, 351(5):1110-1122.

35. Hoofnagle AN, Resing KA, Ahn NG: Protein analysis by hydrogen exchange mass spectrometry. Annu Rev Biophys Biomol Struct 2003, 32:1-25

36. Mann M, Wilm M: Electrospray mass spectrometry for protein characterization. Trends Biochem Sci 1995, 20(6):219-224.

37. Konermann $L$, Tong $X$, Pan $Y$ : Protein structure and dynamics studied by mass spectrometry: H/D exchange, hydroxyl radical labeling, and related approaches. J Mass Spec 2008, 43:1021-1036.

38. Torronen A, Rouvinen J: Structural comparison two major endo-1,4xylanases from Trichoderma reesei,. Biochemistry 1995, 34:847-856.

39. Dai SY, Chalmers MJ, Bruning J, Bramlett KS, Osborne HE, MontroseRafizadeh C, Barr RJ, Wang Y, Wang M, Burris PT, Dodge JA, Griffin PR: Prediction of the tissue-specificity of selective estrogen receptor modulators using a single biochemical method. Proc Natl Acad Sci USA 2007, 105(20):7171-7176.

40. Dai SY, Burris PT, Dodge JA, Montrose-Rafizadeh C, Wang Y, Pascal BD, Chalmers MJ, Griffin PR: Unique ligand binding patterns between estrogen receptor alpha and beta revealed by hydrogen-deuterium exchange. Biochemistry 2009, 48(40):9668-9676.

41. Bai Y, Milne JS, Mayne L, Englander SW: Primary structure effects on peptide group hydrogen exchange. Proteins 1993, 17(1):75-86.

\section{Submit your next manuscript to BioMed Central and take full advantage of:}

- Convenient online submission

- Thorough peer review

- No space constraints or color figure charges

- Immediate publication on acceptance

- Inclusion in PubMed, CAS, Scopus and Google Scholar

- Research which is freely available for redistribution

Submit your manuscript at www.biomedcentral.com/submit
Biomed Central 